\title{
Application of Electrospray Mass Spectrometry and Matrix-Assisted Laser Desorption Ionization Time-of-Flight Mass Spectrometry for Molecular Weight Assignment of Peptides in Complex Mixtures
}

\author{
John R. Perkins, Brian Smith, Richard T. Gallagher, Davis S. Jones, \\ Stephen C. Davis, and Andrew D. Hoffman \\ Kratos Analytical, Urmston, Manchester, United Kingdom
}

\author{
Kenneth B. Tomer
}

National Institute of Environmental Health Sciences, Research Triangle Park, North Carolina, USA

Electrospray mass spectrometry (ES/MS) and matrix-assisted laser desorption ionization time-of-flight mass spectrometry (MALDI/TOF/MS) were used to provide mass spectra from seven elapid snake venoms. Spectral interpretation was much simpler for MALDI/TOF/MS. ES/MS proved more useful for the provision of molecular weight data for very closely related peptides, but suppression of higher molecular weight compounds was seen to occur during flow injection analysis. MALDI/TOF/MS proved useful for providing a complete picture of the venom, but the low resolution led to obscuring of major ions, and the mass accuracy was poorer for known peptides. Suppression also occurred during MALDI/TOF/MS but could be overcome using alternative matrices because the spectra were very dependent on the choice of matrix. ES/MS and MALDI/TOF/MS provide complementary and confirmatory information such that for the analysis of complex peptide mixtures (snake venoms), the use of both techniques is desirable. (J Am Soc Mass Spectrom $1993,4,670-684)$

$\mathrm{B}$ ecause of recent developments in mass spectrometry, there has been an explosion of interest in the molecular weight determination of high molecular weight species. Two of the techniques that have received much attention for the determination of high-mass compounds are electrospray mass spectrometry (ES/MS) and matrix-assisted laser desorption ionization time-of-flight mass spectrometry (MALDI/TOF/MS). Both techniques have proved useful for the characterization of similar classes of compounds, such as peptides, proteins, glycoproteins, and saccharides. Our recent interest is in the analysis of complex peptide mixtures of which snake venoms provide a challenging example.

ES was introduced by Dole et al. [1] in the 1960s when the ionization and multiple charging of macromolecules were demonstrated, although the first reports of ES mass spectra were much later [2]-[5]. The

Address reprint requests to John R. Perkins, Kratos Analytical, Barton Dock Road, Urmston, Manchester, M31 2LD, UK production of multiply charged ions by ES $[6,7]$ and other ionization techniques [8-10] means that the determination of compounds whose molecular weights exceed that of the limits of the mass spectrometer is possible. The applications and mechanisms of ES ionization are discussed in several reviews of atmospheric ionization sources [11-15].

TOF/MS was initially reported by Wiley and McLaren [16] in 1955 and has found numerous applications since then [17]. Interest has recently increased owing to the introduction of MALD by Karas and Hillenkamp [18] and Tanaka et al. [19], which allows the analysis of large molecules in excess of $300 \mathrm{kDa}$ [17]. Spectra are dominated by protonated molecular ions, although slight multiple charging and cluster formation have been seen [20]. Several reviews of MALUI/IUH/MS have been published recently [17, 21-23].

Mambas (Dendroaspis), cobras (Naja, Hemachatus), and coral snakes (Micrurus) are members of the Elapidae family of snakes that also includes kraits 
(Bungarus) and tiger snakes (Notechis) [24]. These snakes all possess venomous bites, and the venoms exist as a complex "cocktail" of chemicals. Dried snake venoms contain 90-95\% small molecular weight protein components that are responsible for the pharmacology and the lethal action [25]. Many of the peptides are not in themselves toxic but act synergistically with others in the venom [26]. The wide range of pharmacologic properties demonstrated by these peptides has meant that interest has been generated in the possible medical applications of snake toxins.

To date, the analysis of snake venoms has mainly been concerned with the extraction of discrete proteins from several Elapidae venoms using gel filtration and ion chromatography to isolate fractions of interest, containing active substances, and with subsequent protein sequencing [24]. Several proteins have been separated and sequenced from the venoms of Dendroaspis jamesoni kaimosae (Jameson's mamba, D. jamesoni), Dendroaspis polylepis polylepis (black mamba, D. polylepis), Dendroaspis augusticeps (eastern green mamba, D. augusticeps), and Hemachatus haemachatus (Ringhals cobra, $H$. haemachatus), but the venom of Micrurus fulvius (eastern coral snake, $M$. fulvius) is totally uncharacterized [24-40].

Protein sequence confirmation and structure determination of toxins isolated from the venoms of $D$. polylepis [41-43] have been performed using ${ }^{1} \mathrm{H}$-nuclear magnetic resonance. Phospholipase $A_{2}$ fractions from the venom of Naja mossambica mossambica (Mozambique spitting cobra, N. mossambica) have been characterized by using ion spray mass spectometry [44], and toxins from the venom of Ophiophagus hannah (Malayan pit viper) have been isolated using preparative scale liquid chromatography (LC) with subsequent molecular weight assignments provided by ES/MS [45]. Methods for the determination of entire snake venoms include reversed-phase high-performance liquid chromatography (HPLC) for characterization of toxins present in the venom of N. mossambica and subsequent application of the method to the study of the venoms from other cobras [46] and on-line capillary electrophoresis (CE)/ES selected ion monitoring mass spectrometry (SIMMS) of venoms from eight different snakes, including those listed previously $[47,48]$. CE/ES/SIMMS provided a rapid means of determination of species present in the venom and proved particularly useful for the separation of peptides of similar molecular weights and those which yielded multiply charged ions of the same mass-to-charge ratio $[47,48]$.

The number of toxins present in snake venoms has been estimated at approximately 30 by conventional methods [49], but CE/ES/SIMMS experiments indicated the presence of approximately 100 toxins in the venom of $D$. polylepis and 60 in the venom of $D$. jamesoni $[47,48]$. We are interested in how much preliminary information about complex peptide mixtures (snake venoms) can be gleaned simply from the mass spectra acquired using ES/MS and MALDI/TOF/MS.

\section{Experimental}

\section{$E S / M S$}

The ES mass spectra were acquired on a Kratos Concept ISQ of EBqQ geometry (Kratos Analytical, Manchester, UK) fitted with an ES ionization source. The ES needle was operated at a potential of $8 \mathrm{kV}$, and the accelerating potential was $4 \mathrm{kV}$. Flow injection analysis of the snake venoms $(1 \mathrm{mg} / \mathrm{mL}$ in distilled water) was performed by placing the sample vial in the well of a stainless steel pressure vessel, described previously [50], and forcing the analyte through a silica column [75- $\mu \mathrm{m}$ inner diameter (i.d.), $150 \mu \mathrm{m}$ outer diameter (o.d.)] by pressurizing with helium. A makeup solution of 50:50 methanol:3\% acetic acid was provided coaxially from a Harvard syringe pump (model 909, Harvard Apparatus, South Natick, MA). Spectra were acquired in the profile mode from $\mathrm{m} / \mathrm{z} 400$ to 2200 at a scan rate of $5 \mathrm{~s}$ /decade. Mass calibration was provided by a mixture of gramicidin $S$ and bovine insulin. The mass resolution was 1000 ( $10 \%$ valley definition). Data acquisition and system control were performed using a Sun SPARC station IPC (Sun Microsystems, Inc., Mountain View, CA).

The $C E$ system was constructed in-house and has been described elsewhere [51]. It utilizes a Glassman HV power supply (Whitehouse Station, NJ) with reversible polarity that was operated at $-22 \mathrm{kV}$. The high-voltage end of the $C E$ capillary is located in a plexiglass box, and the CE ground is the ES needle itself at approximately $8 \mathrm{kV}$, giving an effective voltage drop of approximately $30 \mathrm{kV}$. CE/ES/MS of the venom from $M$. fuloius and Naja melanoleuca (blackand-white cobra, $N$. melanoleuca) was performed using a $1.1-\mathrm{m}$ length of 3-aminopropyltrimethoxysilane derivatized $75-\mu \mathrm{m}$ i.d. fused-silica capillary column with $0.01 \mathrm{M}$ acetic acid buffer ( $\mathrm{pH}$ 3.5). Samples were injected onto the analytical column using a stainless steel pressure vessel [50] or by a timed gravimetric injection.

\section{MALDI/TOF / MS}

Lyophilized snake venom (approximately $1 \mathrm{mg}$ ) was dissolved in distilled water. (Previously, solutions were prepared in $0.1 \%$ trifluoracetic acid, but it was found that the spectral quality of the venoms declined with time because of decomposition.) Prior to acquisition of spectra, $100 \mu \mathrm{L}$ of venom solution was mixed with 100 $\mu \mathrm{L} 0.2 \%$ aqueous trifluoracetic acid; $1 \mu \mathrm{L}$ of this solution was spotted onto a 20-spot stainless steel sample slide, followed by $1 \mu \mathrm{L}$ of sinapinic acid or $\alpha$-cyano-4-hydroxycinnamic acid matrix solution (10 $\mathrm{mg} / \mathrm{mL}$ in 50:50 EtOH $/ \mathrm{H}_{2} \mathrm{O}$ that contained $0.1 \%$ trifluoracetic acid). Mass spectrometry analysis was performed on a Kratos KOMPACT MALDI III LD/TOF mass spectrometer (Kratos Analytical) operating at 20 $\mathrm{kV}$ accelerating potential in the linear mode. Data were 
recorded by using a Sun SPARC station IPC using OPEN LOOK software. Mass spectra were obtained by averaging 50 or 100 shots acquired by rastering across the sample surface or, following this procedure, by sampling on a previously noted "sweet spot" on the sample surface, using a variety of laser powers. Higher laser powers allowed the review of high molecular weight species present in the sample, whereas decreased power provided better discrimination between the lower molecular weight peptides. External mass calibration was provided by the $[\mathrm{M}+\mathrm{H}]^{+}$ions of either bradykinin or bovine insulin and sinapinic acid or by the $[\mathrm{M}+\mathrm{H}]^{+}$ions of bovine insulin and horse heart cytochrome $c$, with extrapolation to the ions of interest. For the venom from $D$. polylepis, where an unambiguous signal with no interference was seen for toxin I $\left(M_{\mathrm{r}} 7132.4\right)$, internal calibration to provide more accurate mass assignment was performed.

\section{Chemicals}

The standard samples of lyophilized snake venoms ( $D$. polylepis, D. jamesoni, D. augusticeps, $H$. haemachatus, $M$. fulvius, N. mossambica, and N. melanoleuca), bradykinin, gramicidin S, bovine insulin, sinapinic acid, a-cyano4-hydroxycinnamic acid, and trifluoracetic acid were obtained from Sigma Chemical Co. (St. Louis, MO). Portions of venoms were dissolved in water that was obtained from a Milli Ro/Milli-Q system (Millipore Corp., Bedford, MA) for ES experiments. The makeup flow contained HPLC-grade acetic acid that was purchased from I. T. Baker Chemical Co. (Phillipsburg, NJ) and the HPLC-grade methanol was obtained from Fisher Scientific (Fair Lawn, NJ). Ethanol was supplied by Aldrich Chemical Co. (Milwaukee, WI).

\section{Results and Discussion}

The ES and MALDI/TOF mass spectra of the snake venoms are illustrated in Figures 1-5, and the information from the spectra is summarized in Tables 1-5. Tables 1-4 are divided into two sections, the first summarizing the sequenced toxins from each venom and the second summarizing unknown peptides. The peptides are listed in order of ascending molecular mass, with the number of charge states identified to assign that molecular mass. The ES mass spectra actually show multiply charged ions, and the molecular masses quoted are based solely on arithmetic calculations where the numbers in parentheses indicate whether any of the ions used to calculate a molecular mass have been used elsewhere [e.g., 4(2) indicates that four ions were found to assign a molecular mass, but two of these were found in common with other peptides]. It is known that ions of different charge states occur at the same mass-to-charge ratio [47], so that allocation of a single mass to more than one molecular mass calculation is possible.
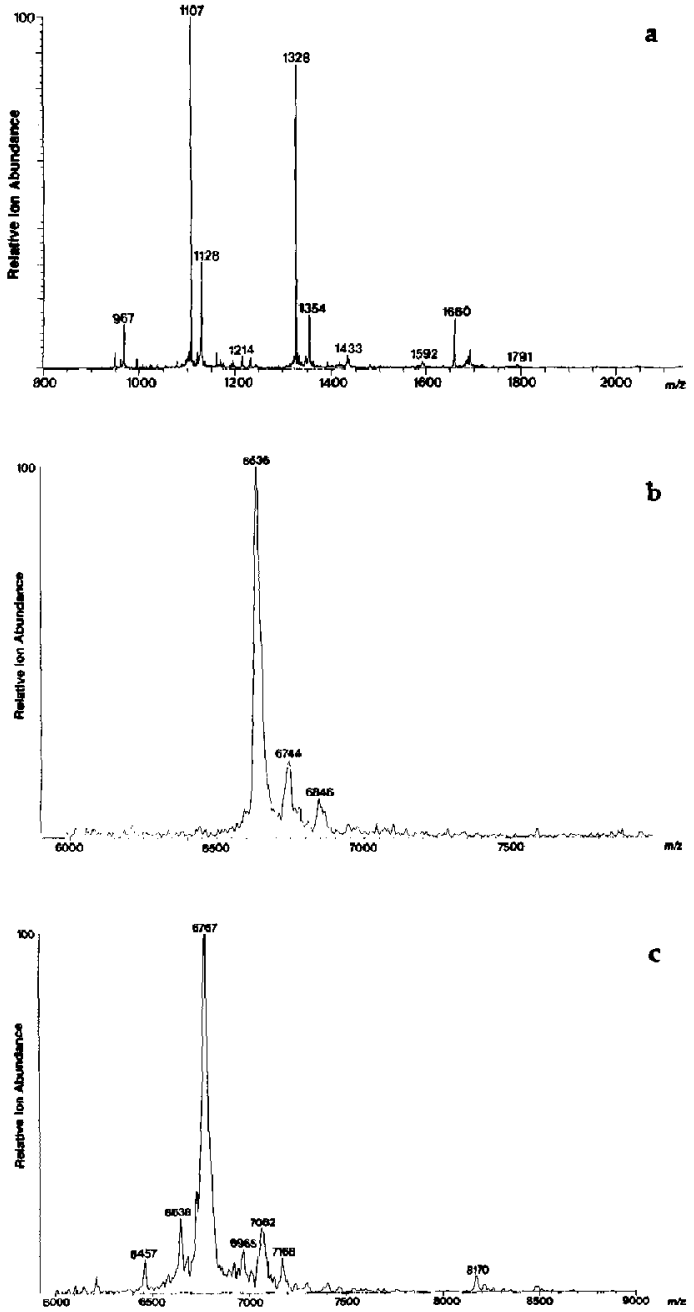

Figure 1. (a) ES mass spectrum of the venom from $D$, jamesoni. (b) Positive ion MALDI/TOF mass spectrum of the venom from D. jamesoni using sinapinic acid matrix. (c) As in (b) but using a-cyano-4-hydroxycinnamic acid matrix.

The accuracies of the molecular masses of sequenced peptides obtained from the ES mass spectra were calculated from the difference between the experimental molecular mass and the mass calculated from the published sequences, and this is expressed as a percentage of the theoretical molecular mass.

The relative abundances of ions seen using each ionization method are listed. ES/MS abundances are listed as a percentage of the summed intensities of the charge states of the most abundant peptide. The CE column indicates whether the peptides were noted in previous CE/ES/SIMMS experiments [47,48].

Both ES/MS and MALDI/TOF/MS can provide useful information about the molecular masses of 

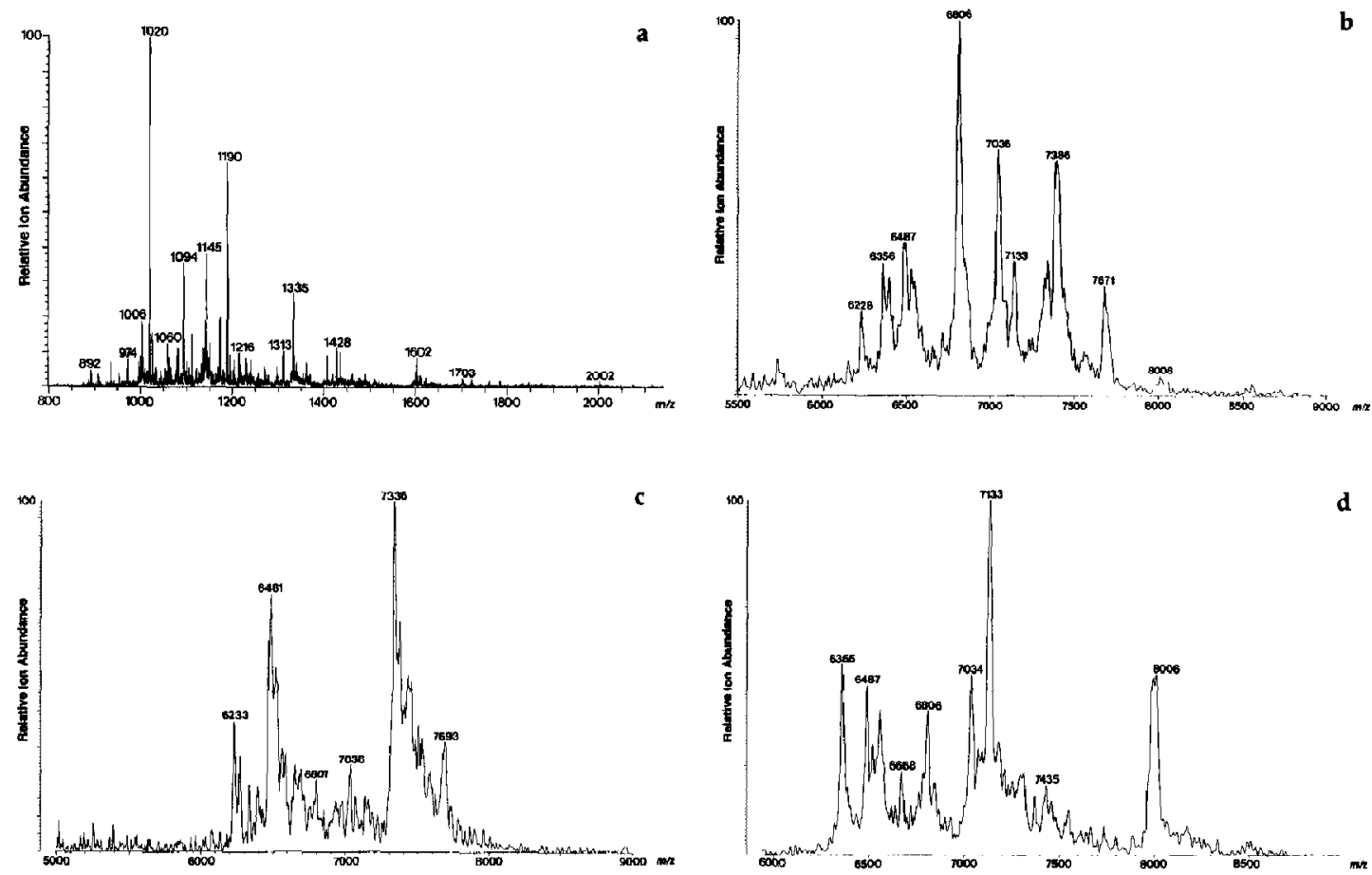

d

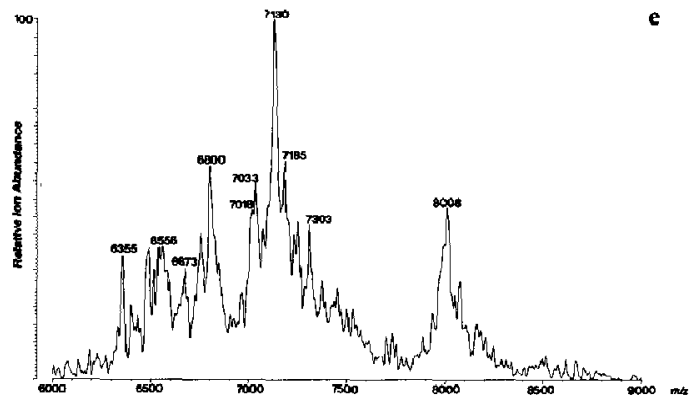

Figure 2. (a) ES mass spectrum of the venum from D. polylepis. (b) Pusitive ion MALDI/TOF mass spectrum of the venom from $D$. polylepis using sinapinic acid matrix. (c) Negative ion MALDI/TOF mass spectrum using sinapinic acid matrix. (d) Positive ion MALDI/TOF mass spectrum using a-cyano-4-hydroxycinnamic acid matrix. (e) Negative ion MALDI/TOF mass spectrum using $\alpha$-cyano-4-hydroxycinnamic acid matrix.

species present within a snake venom. The acquisition of spectra of the snake venoms can be routine using MALDI-TOF-MS, but this is dependent on the matrix identity and the polarity of the accelerating voltage. The acquisition of ES mass spectra was routine, and run-to-run reproducibility was superior to MALDI/ TOF/MS.

Sample throughput is mainly dependent on the ease of spectral interpretation. This is simple for MALDI/TOF/MS because $[\mathrm{M}+\mathrm{H}]^{+}$ions are mainly observed, although some clusters and $[\mathrm{M}+2 \mathrm{H}]^{2+}$ ions were noted. The interpretation of ES mass spectra is, however, more difficult. In general, the toxins with molecular weights in the range 6000-9000 Da show ions ranging from $[\mathrm{M}+8 \mathrm{H}]^{8+}$ (sometimes $[\mathrm{M}+$ $9 \mathrm{H}]^{9+}$ ) to $[\mathrm{M}+4 \mathrm{H}]^{4+}$, whereas the toxins of molecular weights of approximately 13,000 Da show ions ranging from $[\mathrm{M}+10 \mathrm{H}]^{10+}$ to $[\mathrm{M}+7 \mathrm{H}]^{7+}$. Thus, each species will be represented by peaks at several mass-to-charge ratios, and because we know that several peptides are present in each venom, the spectra will contain a large number of apparent peaks. We decided that at least 

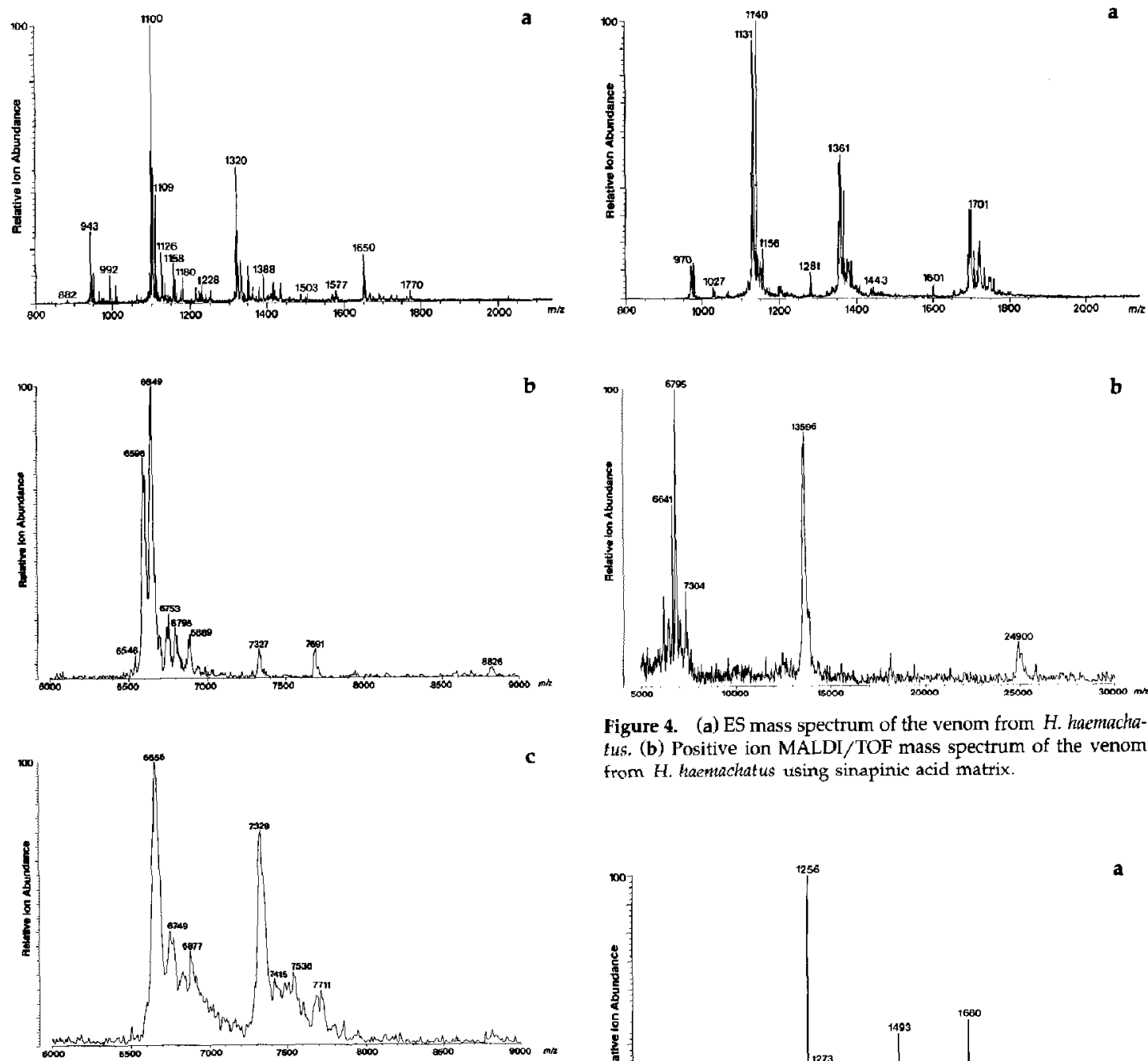

Figure 4. (a) ES mass spectrum of the venom from $H$. haemachatus. (b) Positive ion MALDI/TOF mass spectrum of the venom from $H$. kaemachat us using sinapinic acid matrix

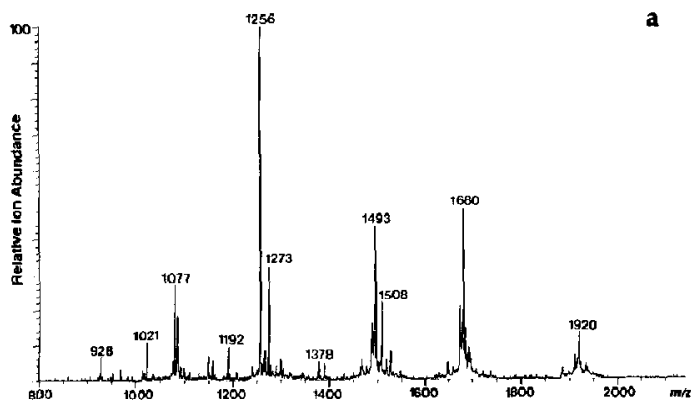

Figure 3. (a) ES mass spectrum of the venom from D. augusticeps. (b) Positive ion and (c) negative ion MALDI/TOF mass spectrum of the venom from $D$. augusticeps using sinapinic acid matrix.

three charge states should be noted to assign molecular weights for any one peptide from the ES spectrum. This inevitably leads to the exclusion of species known from other experimental data but provides a useful control so that spurious peaks are excluded. For comparison, the assignment of molecular weights during CE/MS experiments was based on only two ions because the migration times could be used as an additional discriminatory factor. The venom of $M$. fulvius contained a peptide of molecular weight $6638 \mathrm{Da}$ [48], but the ES mass spectrum revealed only two ions, so definite identification would only be possible after some separation step.

The MALDI/TOF and ES mass spectra of the mambas show that the major proportion of the venom results from lower molecular weight peptides $(m / z=$

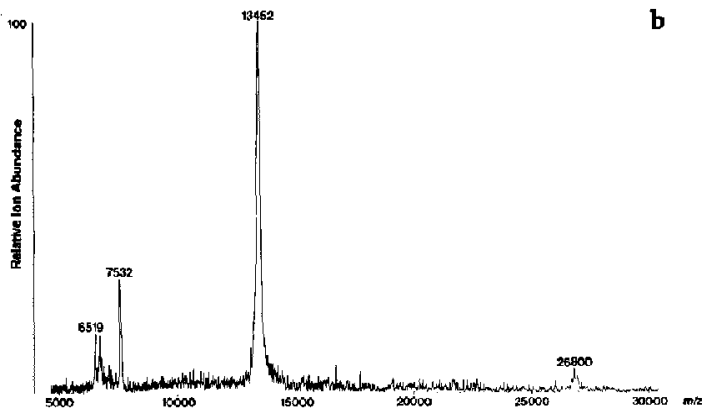

Figure 5. (a) ES mass spectrum of the venom from $M$. fulvius. (b) Positive ion MALDI/TOF mass spectrum of the venom from M. fulvius using a-cyano-4-hydroxycinnamic acid matrix. 
6000-9000) (Figures 1-3, Tables 1-3), whereas a significant portion of the venoms of $H$. haemachatus (Figure 4, Table 4) and $M$. fulvius (Figure 5, Table 5) contain species of molecular masses of approximately 13,000 Da. At higher laser powers, the presence of proteins up to $m / z \quad 50,000$ was noted in the MALDI/TOF mass spectra of $D$, augusticeps, $H$. haemachatus, and $M$. fulvius. For comparison purposes this discussion will concentrate on those peptides of molecular masses of less than 20,000 Da.

Table 1. Cumparison of the peptides ubserved in the ES and MALDI/TOF mass spectra of the venom from D. jamesoni ${ }^{a}$

\begin{tabular}{|c|c|c|c|c|c|c|c|c|c|c|}
\hline \multicolumn{5}{|c|}{ Sequenced toxins } & \multicolumn{5}{|c|}{ Relative ion abundance } & \multirow[b]{2}{*}{$\mathrm{CE}$} \\
\hline Toxin & $M_{\mathrm{r}}$ calc. & $M_{\mathrm{r}}$ ES & $\begin{array}{l}\text { Charge } \\
\text { state }^{\mathrm{a}}\end{array}$ & $\begin{array}{c}\text { Accuracy } \\
(\%)\end{array}$ & $\begin{array}{l}\overline{E S} \\
(\%)\end{array}$ & $\begin{array}{r}+\mathrm{SA} \\
(\%)\end{array}$ & $\begin{array}{r}-5 A \\
(\%)\end{array}$ & $\begin{array}{r}+\mathrm{CHCA} \\
(\%)\end{array}$ & $\begin{array}{r}-\mathrm{CHCA} \\
(\%)\end{array}$ & \\
\hline$\overline{\mathrm{S}_{5} \mathrm{C}_{\text {10 }}}$ & 6513.6 & & & & - & - & - & - & - & - \\
\hline$V_{n} I_{1}$ & 6720.6 & 6719.2 & $4(0)$ & 0.02 & 6 & - & - & 28 & 33 & + \\
\hline $\mathrm{S}_{5} \mathrm{C}_{4}$ & 6764.0 & 6764.4 & $4(1)$ & 0.006 & 34 & 8 & - & 100 & 100 & + \\
\hline $\mathrm{S}_{4} \mathrm{C}_{8}$ & 6960.1 & 6959.7 & $4(0)$ & 0.006 & 5 & 一 & - & 11 & 10 & + \\
\hline$S_{5} C_{1}$ & 7016.0 & 7014.4 & $3(0)$ & 0.02 & 1 & - & - & 6 & - & + \\
\hline $\mathrm{V}_{n} \mathrm{III}_{1}$ & 8175.5 & 8171.7 & $5(3)$ & 0.05 & 3 & - & 一 & 4 & - & + \\
\hline $\mathrm{S}_{2} \mathrm{C}_{4}$ & $13,435.7$ & & & & $\rightarrow$ & 一 & + & - & - & - \\
\hline
\end{tabular}

Other species observed

Relative ion abundance

\begin{tabular}{|c|c|c|c|c|c|c|c|}
\hline \multirow[b]{2}{*}{$M_{r}$} & \multirow[b]{2}{*}{$\begin{array}{c}\text { Charge } \\
\text { state }^{\text {a }}\end{array}$} & & & & & & \multirow[b]{2}{*}{ CE } \\
\hline & & $\begin{array}{l}\text { ES } \\
(\%)\end{array}$ & $\begin{array}{r}+ \text { SA } \\
(\%)\end{array}$ & $\begin{array}{r}- \text { SA } \\
(\%)\end{array}$ & $\begin{array}{r}+\mathrm{CHCA} \\
(\%)\end{array}$ & $\begin{array}{r}-\mathrm{CHCA} \\
|\%\rangle\end{array}$ & \\
\hline$\overline{6220.6}$ & $3(0)$ & 1 & - & - & 4 & - & - \\
\hline 6456.5 & $3(0)$ & 2 & - & - & 9 & - & + \\
\hline 6564.2 & $3(0)$ & 2 & - & - & - & - & + \\
\hline 6577.0 & $3(1)$ & 2 & - & 7 & 5 & - & - \\
\hline 6590.0 & & - & 8 & - & - & 7 & - \\
\hline 6603.1 & $3(0)$ & 4 & - & - & - & 7 & - \\
\hline 6617.6 & $4(1)$ & 5 & - & - & 7 & 一 & - \\
\hline 6634.2 & $4(0)$ & 100 & 100 & 100 & 20 & 18 & - \\
\hline 6655.8 & $4(1)$ & 6 & - & - & - & 12 & - \\
\hline 6674.6 & $4(0)$ & 5 & - & - & 10 & 12 & - \\
\hline 6746.2 & $3(0)$ & 6 & 21 & 47 & - & 91 & + \\
\hline 6786.6 & $4(0)$ & 3 & 8 & - & - & 70 & - \\
\hline 6805.0 & $4(2)$ & 2 & 3 & - & - & $50^{\circ}$ & - \\
\hline 6846.0 & & - & 11 & 11 & 7 & - & - \\
\hline 6855.0 & & - & 8 & - & 7 & 20 & - \\
\hline 6886.0 & & - & - & 6 & 7 & 19 & - \\
\hline 6925.0 & & - & - & - & 8 & 10 & - \\
\hline 6943.0 & & - & 4 & 4 & 7 & 10 & - \\
\hline 7042.6 & $3(0)$ & 2 & 3 & - & - & - & + \\
\hline 7060.2 & $3(0)$ & 1 & - & - & 17 & 8 & - \\
\hline 7089.0 & & - & - & - & 5 & 8 & + \\
\hline 7149.8 & $3(1)$ & 2 & - & - & - & - & + \\
\hline 7168.5 & $4(0)$ & 4 & - & - & 9 & - & + \\
\hline 7385.9 & $4(0)$ & 3 & - & - & 2 & - & - \\
\hline
\end{tabular}

a Charge state refers to the number of multiply charged ions seen to assign any molecular mass, with the numbers in parentheses providing an indication of the number of those multiply charged ions that are also used in the molecular mass assignment of other peptides. + SA, sinapinic acid matrix with $+20-\mathrm{kV}$ accelerating potential; $-\mathrm{SA}$, sinapinic acid matrix with $-20-\mathrm{kV}$ accelerating potential; $+\mathrm{CHCA}$, $\alpha-c y a n a-4$-hydroxycinnamic acid matrix with $+20-\mathrm{kV}$ accelerating potential: $-\mathrm{CHCA}$, $\alpha-c y a n o-4-h y-$ droxycinnamic acid matrix with $-20-k V$ accelerating potential. Species observed using one method but with no corroboration: ES $M S-M, 6539.9$ [3(1), $2 \%$ ]; $M_{r} 6731.5$ [4(0), 5\%]; $M_{r} 7611.6\left[4(1), 2 \%\right.$ ]; $M_{\mathrm{r}}$ $7943.8[3(2), 3 \%] ; M, 8590.3$ [4(0), 3\%]; $M_{\mathrm{r}} 14316.3$ [4(0), 3\%]; $M, 14,338.1$ [3(2), 2\%]. MALDI TOF MS - sinapinic acid (positive ion) $M_{r} 6866$ (7\%); a-cyano-4-hydroxycinnamic acid (positive ion), $M$. $7126(4 \%), M_{r} 7195(4 \%), M_{r} 7241(4 \%), M_{r} 7295(4 \%), M_{r} 7405(4 \%), M_{r} 7460(4 \%), M_{r} 8479(2 \%), M_{r}$ $14,106(2 \%)$; at higher laser powers, $M_{r}, 13,520, M, 14,140 ; \alpha-c y a n o-4$-hydroxycinnam ic acid (negative ion), $M_{\mathrm{r}} 6700(18 \%), M_{\mathrm{r}} 6814(20 \%), M_{\mathrm{r}} 6914(10 \%), M_{\mathrm{r}} 7033(8 \%)$. 
Table 2. Comparison of the peptides observed in the ES and

MALDI/TOF mass spectra of the venom from D. polylepis ${ }^{\text {a }}$

\begin{tabular}{|c|c|c|c|c|c|c|c|c|c|c|}
\hline \multicolumn{3}{|c|}{ Sequenced toxins } & \multirow[b]{2}{*}{$\begin{array}{r}\text { Charge } \\
\text { state }^{a}\end{array}$} & \multirow[b]{2}{*}{$\begin{array}{c}\text { Accuracy } \\
(\%)\end{array}$} & \multicolumn{5}{|c|}{ Relative ion abundance } & \multirow[b]{2}{*}{$\mathrm{CE}$} \\
\hline Toxin & $M_{r}$ calc. & $M_{\mathrm{r}} \mathrm{ES}$ & & & $\begin{array}{l}\text { ES } \\
(\%)\end{array}$ & $\begin{array}{r}+\mathrm{SA} \\
(\%)\end{array}$ & $\begin{array}{r}- \text { SA } \\
(\%)\end{array}$ & $\begin{array}{r}+\mathrm{CHCA} \\
(\%)\end{array}$ & $\begin{array}{r}-\mathrm{CHCA} \\
(\%)\end{array}$ & \\
\hline$\overline{\mathrm{B}}$ & 6469.6 & & & & - & 19 & $56^{b}$ & - & - & - \\
\hline K & 6559.8 & 6558.6 & $4(0)$ & 0.02 & 31 & 30 & $30^{\mathrm{b}}$ & 40 & 37 & + \\
\hline$E$ & 6613.8 & & & & - & - & - & - & - & - \\
\hline C & 6808.9 & 6807.4 & $4(2)$ & 0.02 & 12 & 100 & 21 & 40 & 59 & + \\
\hline$\alpha$ & 6906.9 & & & & - & - & $10^{b}$ & - & 18 & - \\
\hline $\mathrm{FS}_{2}$ & 7018.2 & $7035.1^{\mathrm{c}}$ & $5(1)$ & & 26 & 65 & $25 / 46^{d}$ & 51 & $55 / 47^{d}$ & + \\
\hline 1 & 7132.4 & 7131.7 & $5(0)$ & 0.01 & 100 & 36 & 16 & 100 & 100 & + \\
\hline $\mathrm{CM}-3$ & 7336.4 & & & & - & - & 100 & - & - & - \\
\hline$\delta$ & 7988.4 & 7989.2 & $4(2)$ & 0.01 & 14 & - & 8 & 50 & - & + \\
\hline$\gamma$ & 8033.3 & 8030.7 & $3(0)$ & 0.03 & 10 & - & - & - & - & - \\
\hline A & 8635.2 & 8632.9 & $3(0)$ & 0.03 & 4 & - & - & - & - & - \\
\hline$A^{\prime}$ & 8666.3 & & & & - & - & - & - & - & - \\
\hline
\end{tabular}

Other species observed

Relative ion abundance ${ }^{b}$

\begin{tabular}{|c|c|c|c|c|c|c|c|}
\hline & & & \multirow[b]{2}{*}{$\mathrm{CE}$} \\
\hline$M_{\mathrm{r}}$ & $\begin{array}{l}\text { Charge } \\
\text { state }^{a}\end{array}$ & $\begin{array}{l}\text { ES } \\
(\%)\end{array}$ & $\begin{array}{r}+\mathrm{SA} \\
\langle \%\rangle\end{array}$ & $\begin{array}{r}-S A \\
(\%)\end{array}$ & $\begin{array}{r}+\mathrm{CHCA} \\
(\%)\end{array}$ & $\begin{array}{r}-\mathrm{CHCA} \\
(\%)\end{array}$ & \\
\hline 6074.0 & $3(1)$ & 5 & $\omega$ & 6 & - & - & - \\
\hline 6227.0 & & - & 23 & $23^{h}$ & - & - & - \\
\hline 6356.1 & $4(0)$ & 14 & 36 & - & 54 & 34 & + \\
\hline 6395.0 & $4(3)$ & 6 & 31 & 19 & 14 & 21 & - \\
\hline 6430.0 & & - & - & 12 & 11 & 18 & - \\
\hline 6484.8 & $4(0)$ & 13 & 41 & 74 & 48 & 36 & + \\
\hline 6516.0 & & - & - & 53 & 31 & 30 & - \\
\hline 6537.8 & $3(0)$ & 4 & 34 & $38^{b}$ & $28^{b}$ & 37 & + \\
\hline 6575.0 & & - & - & - & $27^{b}$ & 30 & - \\
\hline 6590.0 & & - & 19 & 28 & - & 27 & - \\
\hline 66350 & & $\ldots$ & & $16^{\mathrm{b}}$ & 14 & - & - \\
\hline 6650.0 & & - & 14 & 25 & - & 21 & - \\
\hline 6670.7 & $4(2)$ & 15 & - & $20^{b}$ & 23 & 31 & + \\
\hline 6690.0 & & - & - & 24 & - & 21 & - \\
\hline 6707.5 & $3(3)$ & 5 & 17 & 16 & 11 & - & - \\
\hline 6723.0 & & - & - & - & - & 21 & + \\
\hline 6760.0 & & - & - & 14 & 18 & 40 & - \\
\hline 6791.5 & $4(0)$ & 7 & - & - & 24 & - & - \\
\hline 6833.4 & $4(1)$ & 7 & - & $11^{\mathrm{b}}$ & 18 & - & - \\
\hline 6847.8 & $3(2)$ & 8 & - & - & 21 & 32 & - \\
\hline 6860.0 & & - & - & 13 & 13 & - & - \\
\hline 6895.0 & & - & 14 & 一 & 11 & - & - \\
\hline 6966.0 & & - & - & $14^{b}$ & - & 24 & - \\
\hline 7005.5 & $4(2)$ & 6 & - & 12 & $15^{b}$ & - & + \\
\hline 7076.6 & $3(0)$ & 7 & 25 & 17 & 30 & 42 & + \\
\hline 7095.0 & & - & - & $11^{b}$ & 28 & - & - \\
\hline 7173.3 & $5(1)$ & 18 & - & - & 32 & 49 & + \\
\hline 7185.0 & & - & - & 12 & - & 60 & - \\
\hline 7216.1 & $4(0)$ & 3 & 16 & $8^{b}$ & 24 & - & - \\
\hline 7230.2 & $3(2)$ & 5 & 16 & $10^{b}$ & 20 & 40 & - \\
\hline 7251.0 & & - & - & 10 & 21 & 44 & - \\
\hline 7268.0 & & - & - & 10 & - & 31 & - \\
\hline
\end{tabular}


Table 2. (Continued)

\begin{tabular}{|c|c|c|c|c|c|c|c|}
\hline \multicolumn{2}{|c|}{ Other species observed } & \multicolumn{5}{|c|}{ Relative ion abundance } & \multirow[b]{2}{*}{ CE } \\
\hline$M_{\mathrm{r}}$ & $\begin{array}{l}\text { Charge } \\
\text { state }^{a}\end{array}$ & $\begin{array}{l}E S \\
(\%)\end{array}$ & $\begin{array}{r}+S A \\
(\%)\end{array}$ & $\begin{array}{r}-S A \\
(\%)\end{array}$ & $\begin{array}{r}+\mathrm{CHCA} \\
(\%)\end{array}$ & $\begin{array}{r}-\mathrm{CHCA} \\
(\%)\end{array}$ & \\
\hline 7289.6 & $4(1)$ & 5 & - & - & 18 & 26 & - \\
\hline 7307.2 & $5(1)$ & 10 & - & - & 23 & 43 & - \\
\hline 7320.0 & & - & 32 & - & 23 & 一 & - \\
\hline 7350.6 & $3(1)$ & 3 & 36 & $58^{\mathrm{b}}$ & - & - & - \\
\hline 7378.6 & $4(2)$ & 5 & 62 & 66 & 16 & 28 & - \\
\hline 7439.3 & $5(0)$ & 13 & - & $46^{\mathbf{b}}$ & 19 & - & - \\
\hline 7462.0 & & - & - & 48 & 15 & 25 & - \\
\hline 7502.0 & & - & - & 36 & - & 19 & - \\
\hline 7543.7 & $5(2)$ & 6 & 12 & $27^{\mathrm{b}}$ & 12 & -- & - \\
\hline 7670.8 & $4(2)$ & 6 & 29 & $28^{b}$ & 16 & - & - \\
\hline 7973.9 & $5(0)$ & 20 & - & - & - & - & + \\
\hline 8004.4 & $5(2)$ & 41 & 5 & 5 & 51 & 48 & + \\
\hline 8045.2 & $4(2)$ & 19 & - & - & $21^{\mathrm{b}}$ & 24 & - \\
\hline 8506.1 & $4(2)$ & 10 & - & - & 8 & - & - \\
\hline
\end{tabular}

See Table 1 for explanation of charge state, $+S A_{r}-S A_{1}+C H C A$, and - CHCA. Species observed using one method but with no corroboration; ES $M S-M_{r} 8403.2\left[3(1), 3 \%\right.$ ]; $M_{r} 8433.8[3(3), 5 \%] ; M_{r}$ 8603.8 [4(2), 8\%]. MALDI/TOF MS - sinapinic acid (positive ion), $M_{\text {, } 8549}$ (3\%); sinapinic acid (negative ion) $M_{\mathrm{r}} 6233(37 \%) . M_{\mathrm{r}} 6268(28 \%), M_{\mathrm{r}} 6337(19 \%) . M_{\mathrm{r}} 6368(9 \%), M_{\mathrm{r}} 6387\left(17 \%\right.$ ) $M_{\mathrm{r}}$ $6415(13 \%), M_{\mathrm{r}} 6565(31 \%), M_{\mathrm{r}} 6604(14 \% *), M_{\mathrm{r}} 6747(13 \% *), M_{\mathrm{r}} 6784(15 \% *), M_{\mathrm{r}} 6820\left(11 \%^{*}\right), M_{\mathrm{r}}^{\mathrm{r}}$ $6873(7 \%), M, 6934(15 \%), M, 6948\left(13^{*}\right), M, 6978(15 \%), M, 7108(10 \%), M, 7160(15 \%), M_{r}, 7222$ (11\%). $M_{\mathrm{r}} 7408(41 \%), M_{\mathrm{r}} 7431(50 \%), M_{\mathrm{r}} 7485(32 \%), M_{\mathrm{r}} 7525(32 \%), M_{\mathrm{r}} 7582(22 \%), M_{\mathrm{r}} 7599$ $\left.(18 \% *), M_{r} 7623(16 \%), M_{r} 7693(32 \%), M_{r} 7733(12 \%), M_{r}, 7781(10 \%), M_{r}, 7804(7 \% *), M_{r} 783\right\urcorner(5 \%)$. $M_{r} 7866(8 \%), M_{r} 7900$ (8\%); $\alpha$-cyano-4-hydroxycinnamic acid (positive ion), $M_{r} 6380$ (19\%), $M_{r} 6456$ $(10 \%), M, 8055(18 \%), M, 8174(16 \%)$; a-cyano-4-hydroxycinnamic acid (negative ion), $M, 7532(19 \%)$, $M, 7940(37 \%), M, 8075(26 \%), M_{r} 8150(15 \%)$.

'Ion seen as shoulder of main peak.

- Theoretıcal molecular weight of toxin $\mathrm{FS}_{2}$ is $/ 018$. It has been identitied as the third most abundant toxin in the venom of $D$. polylepis, yet little experimental evidence exists of a major component of this molecular mass. In all experiments we have noted an abundant species of molecular mass 7035 (except in negative ion MALDI/TOF /MS using $\alpha$-cyano-4-hydroxycinnamic acid matrix) and have postulated that this species is toxin $\mathrm{FS}_{2}$.

Using $\alpha$-cyano-4-hydroxycinnam ic acid matrix the ion of $47 \%$ relative ion abundance occurred at $\mathrm{m} / \mathrm{z} 7018$ and was sean as a shoulder on the peak resulting from the protonated molecular ion of the peptide of $m / 27035$. Using sinapinic acid matrix, an ion of $m / 27023$ (relative abundance $=16 \%$ ) was noted as a shoulder on the ion of $m / 27036$ (relative abundance $=25 \%$ ).

\section{Mass Accuracy and Resolution}

Although we looked at the full venoms (up to 100 peptides in each), it is possible to identify the presence of peptides with molecular masses that match the published sequences for several species. Of the 24 sequenced peptides for which molecular masses could be assigned from the ES mass spectrum, the mass accuracy mainly ranges from $0.006 \%$ (toxins $\mathrm{S}_{5} \mathrm{C}_{4}$ and $\mathrm{S}_{4} \mathrm{C}_{8}, D$. jamesoni) to $0.05 \%$ (toxins $\mathrm{V}_{n} \mathrm{III}_{1}$, D. jamesoni;

Table 3. Comparison of the peptides observed in the ES and MALDI/TOF mass spectra of the venom from D. augusticeps

\begin{tabular}{|c|c|c|c|c|c|c|c|c|c|c|}
\hline \multicolumn{3}{|c|}{ Sequenced toxins } & \multirow[b]{2}{*}{$\begin{array}{l}\text { Charge } \\
\text { state }^{\mathrm{a}}\end{array}$} & \multirow[b]{2}{*}{$\begin{array}{c}\text { Accuracy } \\
\text { (\%) }\end{array}$} & \multicolumn{5}{|c|}{ Relative ion abundance } & \multirow[b]{2}{*}{$\mathrm{CE}$} \\
\hline Toxin & $M_{\mathrm{r}}$ calc. & $M_{\mathrm{r}}$ ES & & & $\begin{array}{l}\mathrm{ES} \\
(\%)\end{array}$ & $\begin{array}{r}+5 A \\
(\%)\end{array}$ & $\begin{array}{r}-5 A \\
|\%|\end{array}$ & $\begin{array}{r}+\mathrm{CHCA} \\
(\%)\end{array}$ & $\begin{array}{r}-\mathrm{CHCA} \\
(\%)\end{array}$ & \\
\hline$\overline{C_{13} S_{1} \bar{C}_{1}}$ & 6539.9 & 6538.2 & $4(0)$ & 0.03 & 4 & 7 & - & 一 & 16 & - \\
\hline$c_{13} s_{1} c_{3}$ & 6567.8 & 6564.4 & $4(1)$ & 0.05 & 10 & $5^{b}$ & - & $7^{b}$ & $24^{b}$ & - \\
\hline$F_{8}$ & 6583.9 & 6579.2 & $4(1)$ & 0.07 & 8 & - & - & $94^{b}$ & - & - \\
\hline Fasciculin 2 & 6749.8 & 6748.0 & $4|1\rangle$ & 0.03 & 15 & 21 & 40 & $19^{b}$ & 30 & + \\
\hline Fasciculin 1 & $6796.6^{\circ}$ & & $4(0)$ & & 8 & 17 & - & 14 & 13 & + \\
\hline $\mathrm{C}_{10} \mathrm{~S}_{2} \mathrm{C}_{2}$ & 6810.0 & & & & - & 10 & $24^{b}$ & 8 & 12 & - \\
\hline$\alpha$-Dendrotoxin & 7048.2 & 7046.9 & $4(0)$ & 0.02 & 6 & - & 11 & - & 10 & + \\
\hline Muscarinic 2 & 7076.1 & 7074.0 & $4(0)$ & 0.03 & 12 & - & 9 & 9 & 7 & + \\
\hline $\mathrm{C}_{8} \mathrm{~S}_{2}$ & & & & & - & - & - & - & - & - \\
\hline$C_{9} S_{3}$ & & & & & -- & - & - & - & - & - \\
\hline
\end{tabular}


Table 3. (Continued)

\begin{tabular}{|c|c|c|c|c|c|c|c|}
\hline \multicolumn{2}{|c|}{ Other species observed } & \multicolumn{5}{|c|}{ Relative ion abundance } & \multirow[b]{2}{*}{ CE } \\
\hline$M_{\mathrm{r}}$ & $\begin{array}{l}\text { Charge } \\
\text { state }^{a}\end{array}$ & $\begin{array}{l}\text { ES } \\
\langle \%\rangle\end{array}$ & $\begin{array}{r}+S A \\
(\%)\end{array}$ & $\begin{array}{r}-S A \\
(\%)\end{array}$ & $\begin{array}{r}+\mathrm{CHCA} \\
(\%)\end{array}$ & $\begin{array}{r}-\mathrm{CHCA} \\
(\%)\end{array}$ & \\
\hline 6555.0 & & - & - & - & 10 & 24 & - \\
\hline 6596.3 & $4(0)$ & 100 & 76 & $16^{\mathrm{b}}$ & 100 & $100^{\circ}$ & + \\
\hline 6609.9 & $4(0)$ & 51 & $70^{b}$ & - & $16^{b}$ & $100^{d}$ & + \\
\hline 6637.3 & $4(0)$ & 7 & - & - & 23 & - & - \\
\hline 6651.6 & $4(0)$ & 40 & 100 & 100 & 22 & 34 & + \\
\hline 6670.0 & & - & $36^{\mathrm{b}}$ & - & 4 & $21^{\mathrm{b}}$ & - \\
\hline 6693.1 & $3(1)$ & 4 & 15 & - & 9 & - & - \\
\hline 6704.0 & & - & $14^{b}$ & - & 15 & 17 & - \\
\hline 6712.0 & & - & 10 & - & - & $13^{b}$ & - \\
\hline 6736.0 & & - & 17 & $30^{b}$ & 32 & - & - \\
\hline 6763.0 & $4(1)$ & 5 & $13^{b}$ & - & 12 & - & - \\
\hline 6773.0 & & - & 8 & 38 & - & - & - \\
\hline 6804.0 & & - & $14^{\mathrm{b}}$ & - & - & 12 & - \\
\hline 6824.5 & $3(0)$ & 4 & - & 26 & 7 & $8^{b}$ & - \\
\hline 6840.0 & & - & 6 & 24 & 8 & 5 & - \\
\hline 6867.0 & & - & $6^{\mathrm{b}}$ & - & 6 & 4 & - \\
\hline 6877.0 & & $\cdots$ & 13 & 33 & - & - & - \\
\hline 6886.1 & $4(1)$ & 5 & 14 & - & 2 & 9 & - \\
\hline 6905.0 & & - & - & 24 & $7^{b}$ & 10 & - \\
\hline 6927.0 & & - & $3^{b}$ & - & 10 & - & - \\
\hline 6939.6 & $4(0)$ & 20 & 4 & 19 & 一 & 12 & 1 \\
\hline 6978.2 & $3(0)$ & 4 & 3 & 16 & 6 & - & - \\
\hline 6991.0 & & -- & - & - & 7 & $10^{\mathrm{b}}$ & - \\
\hline 6998.0 & & - & - & 13 & 8 & 12 & - \\
\hline 7017.0 & & - & - & 14 & - & 10 & - \\
\hline 7028.0 & $4(1)$ & 3 & 2 & - & $4^{b}$ & $10^{\mathrm{b}}$ & - \\
\hline 7038.0 & & - & 2 & $10^{b}$ & 7 & 11 & - \\
\hline 7090.0 & $4(0)$ & 4 & - & - & 7 & - & - \\
\hline 7107.0 & & -- & - & 10 & 3 & - & - \\
\hline 7161.0 & & - & 一 & 9 & 8 & - & - \\
\hline 7201.0 & & - & - & 7 & 3 & 一 & - \\
\hline 7280.8 & $4(0)$ & 4 & 2 & - & 3 & - & - \\
\hline 7316.0 & & - & - & - & $2^{b}$ & 4 & - \\
\hline 7332.3 & $3(0)$ & 4 & 10 & 75 & - & - & - \\
\hline 7425.4 & $4(0)$ & 4 & - & $21^{b}$ & - & 5 & - \\
\hline 7449.0 & & - & 一 & $19^{b}$ & - & 5 & - \\
\hline 7487.6 & $4(0)$ & 3 & - & 22 & - & - & - \\
\hline 7508.9 & $4(0)$ & 4 & - & 22 & - & - & - \\
\hline 7571.0 & & - & - & $16^{b}$ & - & 3 & - \\
\hline 7685.0 & & - & 9 & 17 & 一 & - & - \\
\hline 7710.0 & & - & 3 & 20 & - & - & - \\
\hline
\end{tabular}

${ }^{a}$ See Table 1 for explanation of charge state, +SA, - SA, +CHCA, and - CHCA. Species observed using one method but with no corroboration: ES MS - $M, 6848.5$ [3(0), 4\%]; $M, 7875.7$ [3(2). 8\%]: $M$, $7891.1[3(2), 4 \%], M, 8032.3[3(3), 2 \%]$. MALDI $\tau$ OF $/ M S$ ( ${ }^{*}$ indicates ion seen as shoulder on major peak) - sinapinic acid (positive ion), $M_{r} 6514(2 \%), M_{r} 6616(49 \% *), M_{r} 6627(30 \% \star), M_{r} 6815(9 \%), M_{r}$ $6858(4 \% *), M_{r} 7216(2 \%), M_{r} 8825(4 \%)$; sinapinic acid (negative ion), $M_{r} 6502(6 \%), M_{r} 7179(7 \%)$. $M, 7234(8 \%), M_{,} 7257(9 \%), M_{r} 7338(7 \% *), M_{r} 7415(23 \%), M_{r} 7536(26 \%), M_{r} 7601(15 \%), M_{t} 7745$ (9\%), $M_{r} 7799$ (8\%), $M_{r} 7857$ (9\%), $M_{r} 7945$ (5\%); $\alpha$-cyano-4-hydroxycinnamic acid (positive jon), $M$. $6663(7 \%), M_{\mathrm{r}} 6783(15 \%), M_{\mathrm{r}} 7189(3 \%), M_{\mathrm{r}} 7300(3 \%), M_{\mathrm{r}} 7554(5 \%)$; -cyano-4-hydroxycinnamic acid (negative ion), $M_{r} 6727(24 \%), M_{r} 6953(9 \%), M_{r} 7367(5 \%)$.

Ion seen as shoulder on major peak.

${ }^{c}$ Fasciculin 1 is noted in the literature as having the same sequence as fasciculin 2 except for replacement of an asparagine/aspartic acid by tyrosine [36]. ES /MS can confirm this to be the case but when looking at the entire venom, the mass accuracy is insufficient to determine whether asparagine or aspartic acid is the substituted amino acid.

"The base peak in the negative ion MALDI/TOF mass spectrum using a-cyano-4-hydroxycinnamic acid matrix occurred at $\mathrm{m} / z 6603$. This was thought to result from the merging of the signals due to the $\left[\mathrm{M}+\mathrm{H}^{+}\right.$ions of the peptides of molecular masses 6596 and 6610 . 
Table 4. Comparison of the peptides observed in the ES and MALDI/TOF mass spectra of the venom from $H$. haemachatus ${ }^{\text {a }}$

\begin{tabular}{|c|c|c|c|c|c|c|c|c|c|c|}
\hline \multicolumn{3}{|c|}{ Sequenced toxins } & \multirow[b]{2}{*}{$\begin{array}{l}\text { Charge } \\
\text { state }^{\mathrm{a}}\end{array}$} & \multirow[b]{2}{*}{$\begin{array}{c}\text { Accuracy } \\
(\%)\end{array}$} & \multicolumn{5}{|c|}{ Relative ion abundance } & \multirow[b]{2}{*}{ CE } \\
\hline Toxin & $M_{\mathrm{r}}$ calc. & $M_{\mathrm{r}}$ ES & & & $\begin{array}{l}\text { ES } \\
(\%)\end{array}$ & $\begin{array}{r}+5 A \\
(\%)\end{array}$ & $\begin{array}{r}-5 A \\
(\%)\end{array}$ & $\begin{array}{r}+\mathrm{CHCA} \\
(\%)\end{array}$ & $\begin{array}{r}-\mathrm{CHCA} \\
(\%)\end{array}$ & \\
\hline $\mathrm{HHVII}$ & 6401.4 & 6400.4 & $3(1)$ & 0.02 & 5 & 20 & - & 17 & - & - \\
\hline 11 & 6784.4 & 6782.5 & $4(0)$ & 0.03 & 96 & $83^{b}$ & - & 100 & 100 & + \\
\hline $12 \mathrm{~A}$ & 6792.4 & 6789.2 & $3(0)$ & 0.05 & 16 & 100 & 一 & - & - & - \\
\hline $11 \mathrm{~A}$ & 6800.4 & 6798.2 & $4(0)$ & 0.03 & 100 & - & 100 & - & $98^{b}$ & + \\
\hline IV & 6823.7 & & & & - & - & - & - & 94 & - \\
\hline II & 6829.8 & & & & - & - & 一 & - & - & - \\
\hline $12 \mathrm{~B}$ & 6836.6 & 6835.1 & $4(0)$ & 0.02 & 73 & $60^{b}$ & - & 71 & - & + \\
\hline $9 B$ & 7185.7 & 7183.9 & $4(0)$ & 0.03 & 7 & - & - & $35^{b}$ & 22 & + \\
\hline 9BB & 7213.7 & 7211.6 & $4(0)$ & 0.03 & 5 & - & - & 32 & 17 & - \\
\hline Phospholipase & $13,505.3$ & & & & - & & 2 & 3 & & - \\
\hline
\end{tabular}
A DE-1

Other species observed

\begin{tabular}{|c|c|c|c|c|c|c|c|}
\hline \multicolumn{2}{|c|}{ Other species observed } & \multicolumn{5}{|c|}{ Relative ion abundance } & \multirow[b]{2}{*}{ CE } \\
\hline$M_{\mathrm{r}}$ & $\begin{array}{l}\text { Charge } \\
\text { state }^{\circ}\end{array}$ & $\begin{array}{l}E S \\
(\%)\end{array}$ & $\begin{array}{r}+ \text { SA } \\
(\%)\end{array}$ & $\begin{array}{r}-\mathrm{SA} \\
(\%)\end{array}$ & $\begin{array}{r}+\mathrm{CHCA} \\
(\%)\end{array}$ & $\begin{array}{r}-\mathrm{CHCA} \\
(\%)\end{array}$ & \\
\hline 6391.0 & & - & - & 13 & - & 16 & - \\
\hline 6419.9 & $3(0)$ & 2 & 22 & 13 & 11 & - & - \\
\hline 6580.0 & & -- & 18 & 6 & $12^{b}$ & $10^{b}$ & - \\
\hline 6603.0 & & - & - & 8 & 22 & $16^{\circ}$ & - \\
\hline 6621.4 & $3(0)$ & 4 & - & - & $15^{\mathrm{b}}$ & 18 & - \\
\hline 6641.0 & & - & 60 & 36 & 32 & - & - \\
\hline 6674.8 & $3(0)$ & 2 & - & - & 10 & $12^{b}$ & - \\
\hline 6687.6 & $4(0)$ & 6 & 一 & - & 10 & $16^{b}$ & - \\
\hline 6706.2 & $3(0)$ & 5 & - & - & 40 & - & - \\
\hline 6716.8 & $3(0)$ & 3 & - & $10^{b}$ & 15 & 23 & - \\
\hline 6726.2 & $4(0)$ & 4 & - & $15^{\mathrm{b}}$ & - & - & - \\
\hline 6737.2 & $3(0)$ & 8 & - & - & $33^{b}$ & - & - \\
\hline 6748.4 & $4(0)$ & 8 & 32 & $18^{b}$ & - & $44^{b}$ & - \\
\hline 6768.0 & $4(0)$ & 47 & $84^{b}$ & $38^{b}$ & $93^{b}$ & - & - \\
\hline 6814.6 & $4(0)$ & 23 & 82 & - & 93 & - & - \\
\hline 6851.7 & $4(0)$ & 13 & 46 & $50^{\mathrm{h}}$ & 43 & - & + \\
\hline 6866.3 & $3(0)$ & 12 & - & $40^{b}$ & 一 & $38^{c}$ & - \\
\hline 6879.7 & $4(0)$ & 23 & 26 & - & 42 & $38^{\circ}$ & - \\
\hline 6889.2 & $4(0)$ & 11 & - & $45^{b}$ & - & - & - \\
\hline 6899.7 & $4(0)$ & 25 & 21 & 52 & - & 26 & - \\
\hline 6934.1 & $4(0)$ & 23 & - & 24 & - & 26 & + \\
\hline 6962.0 & & - & - & $13^{b}$ & 15 & 12 & - \\
\hline 6994.9 & $3(0)$ & 8 & - & 16 & - & - & - \\
\hline 7000.0 & & - & 18 & - & 10 & 10 & - \\
\hline 7019.0 & & - & - & 20 & - & 9 & - \\
\hline 7031.4 & $3(0)$ & 8 & 25 & - & 7 & - & - \\
\hline 7055.0 & & - & 24 & 16 & - & - & - \\
\hline 7114.0 & & - & - & 16 & 10 & - & - \\
\hline 7164.0 & & 一 & - & 11 & 37 & 17 & - \\
\hline 7205.0 & & - & - & 10 & - & 17 & - \\
\hline 7226.0 & & - & - & 10 & - & 13 & - \\
\hline 7243.0 & & - & - & 10 & - & 10 & - \\
\hline 7294.0 & & - & - & 13 & 13 & - & - \\
\hline 7415.0 & & - & - & 20 & 一 & 10 & - \\
\hline
\end{tabular}


Table 4. (Continued)

\begin{tabular}{|c|c|c|c|c|c|c|c|}
\hline \multicolumn{2}{|c|}{ Other species observed } & \multicolumn{5}{|c|}{ Relative ion abundance } & \multirow[b]{2}{*}{ CE } \\
\hline$M_{\mathrm{r}}$ & $\begin{array}{c}\text { Charge } \\
\text { state }^{\mathrm{a}}\end{array}$ & $\begin{array}{l}\overline{E S} \\
(\%)\end{array}$ & $\begin{array}{r}+\mathrm{SA} \\
(\%) \\
\end{array}$ & $\begin{array}{r}-\mathrm{SA} \\
|\%|\end{array}$ & $\begin{array}{r}+\mathrm{CHCA} \\
(\%) \\
\end{array}$ & $\begin{array}{r}-\mathrm{CHCA} \\
(\%) \\
\end{array}$ & \\
\hline $13,520.0$ & & - & - & - & $+^{\circ}$ & $+c$ & - \\
\hline $13,560.0$ & & - & 80 & 2 & - & 2 & - \\
\hline $13,600.0$ & & - & 85 & - & $+c$ & - & - \\
\hline $13,620.0$ & & - & 80 & 2 & 一 & - & - \\
\hline $13,760.0$ & & - & 30 & - & $+c$ & - & - \\
\hline
\end{tabular}

a See Table 1 for explanation of charge state, +SA, - SA, + CHCA, and - CHCA. Species observed using a single method but with no corroboration: ES $/ M S-M_{r} 6698.7[4(0), 5 \%] ; M_{\mathrm{r}} 7683.7[3(1), 4 \%]$. MALDI $/$ TOF $/ M S-$ sinapinic acid (positive ion), $M_{r} 6159(30 \%), M, 7304(32 \%), M_{r} 7381(18 \%), M_{r}$ $7594(12 \%), M_{r} 13,540(76 \%), M_{r} 13.829(26 \%), M_{r} 24,901$ (15\%); sinapinic acid (negative ion), $M_{r}$ $5869(22 \%), M_{r} 6483(8 \%), M_{r} 6501(8 \%), M_{r} 6523(8 \%), M_{r} 7092(15 \%), M_{r} 7320(20 \%), M_{r} 7481$ (10\%), $M, 13,720(2 \%) ; \alpha$-cyano-4-hydroxycinnamic acid (positive ion), $M_{r} 6383(21 \%), M_{r} 6610(21 \%)$. $M_{\mathrm{r}} 7258$ (16\%), $M_{\mathrm{r}} 7365(11 \%), M_{\mathrm{r}} 13,480$ (3\%); at higher laser powers, $M_{\mathrm{r}} 13,460, M_{\mathrm{r}} 13,655, M_{\mathrm{r}}$ 13,700 .

${ }_{\alpha}$-cyano-4-hydroxycinnamic acid (negative ion), $M_{r} 6351$ (10\%), $M_{r} 6376(12 \%), M_{r} 6514(16 \%), M_{r}$ $7045(9 \%), M_{r} 7144(12 \%), M_{r} / 352(10 \%), M_{r} / 432$ (9\%). lons are seen as shoulders on major peaks.

"Seen at higher laser powers than that of the spectrum on which relative ion abundances are based.

$\mathrm{C}_{13} \mathrm{~S}_{1} \mathrm{C}_{3}, D$. augusticeps; and $12 \mathrm{~A}, H$. haemachatus), although toxin $F_{8}$ in the venom of $D$. augusticeps (Figure 3 , Table 3 ) shows a mass accuracy of only $0.07 \%$. This compares with a mass accuracy of $0.1 \%$ obtained with external calibration for MALDI/TOF/MS. Where multiply charged species of several components coincide at the same mass in the ES mass spectrum, accurate assignment will be more difficult.

For toxins 11,11A, and $12 \mathrm{~A}$ in the venom of $H$. haemachatus whose molecular masses differ from one another by $8 \mathrm{u}$, we can still assign molecular weights with greater than $0.05 \%$ mass accuracy (Figure 4, Table 4). For most cases where both MALDI/TOF/MS and ES/MS indicate the presence of the same peptide, the calculated molecular weight from the ES mass spec trum is assumed to be the more correct assignment.

The low resolution of MALDI/TOF/MS means that baseline resolution of peptides with closely related molecular masses can be difficult. The ES mass spectrum of D. jamesoni (Figure 1, Table 1) identifies the presence of several peptides that have molecular masses between 6540 and $6800 \mathrm{Da}$, particularly of approximately 6630 and $6750 \mathrm{Da}$. The MALDI/TOF mass spectra can only unequivocally identify the major ions, whereas the less abundant species either will be noted as shoulders on the major peaks or will be totally obscured by the more abundant ions. Species that are seen as shoulders on larger peaks in the MALDI/TOF mass spectra are noted in Tables 1-5.

A more extreme example of the inability of MALDI/TOF/MS to differentiate closely related molecular masses can be seen in the lower mass region of the spectra of the venom from $H$. haemachatus (Figure 4, Table 4), where most of the abundant peptides occur between 6740 and $6940 \mathrm{Da}$. In this region of the ES mass spectrum we noted 13 peptides, including the sequenced toxins $11,11 \mathrm{~A}, 12 \mathrm{~A}$, and $12 \mathrm{~B}$. In comparison, the best MALDI/TOF/MS results were obtained using sinapinic acid in the positive ion mode, where essentially six major peptides were noted, including toxin 12A $\left(M_{\mathrm{r}} 6792.4\right.$, seen as $\left.m / z 6795\right)$ and the unsequenced peptides of molecular weights 6750,6814 , 6853,6880 , and $6900 \mathrm{Da}$, together with three other peptides noted as shoulders on the main peaks.

Several of these peptides have been isolated and sequenced, and it was found that ES/MS alone could not indicate all of the species present. Toxins $\Pi, \mathrm{IV}$, and 12B, with molecular weights of $6829.8,6823.7$, and 6836.6 Da, respectively, were not resolved. Previous CE/MS experiments indicated the response of toxin $12 \mathrm{~B}$ to be approximately 12 times that of toxin $\mathrm{II}$, with toxin IV not seen at all [48]. The presence of only one peak in the ES spectrum can be explained by the large response seen for toxin $12 \mathrm{~B}$, overwhelming that of the more minor toxin II. For these peptides, a separation stage prior to characterization by mass spectrometry is vital.

The base peaks in the MALDI/TOF mass spectra acquired for the venom of $M$. fulvius arise from peptides with molecular masses between 13,350 and 13,500. Unfortunately, the reduced resolution of MALDI/TOF/MS does not allow definite identification of the less abundant peptides in this molecular weight region because they are only noted as shoulders on the main peak. In this case, the use of ES/MS is ideal for confirmation of these less abundant peptides (Figure 5, Table 5).

For venoms that contain a large number of closely related peptides, MALDI/TOF/MS provides a useful indication of the approximate molecular weights of groups of ions, whereas ES/MS can be used to provide extra accuracy for characterization of the group.

\section{Ion Suppression in the ES Mass Spectrum}

Reports in the literature state that cobra venoms contain a phospholipase $A_{2}$ fraction with molecular masses of approximately 13,000 [46]. The venom of D. augusti- 
Table 5. Comparison of the peptides observed in the ES and MALDI/TOF mass spectra of the venom from $M$. fulvius ${ }^{\mathrm{a}}$

\begin{tabular}{|c|c|c|c|c|c|c|c|}
\hline \multirow[b]{2}{*}{$\begin{array}{l}\text { Species observed } \\
\text { in spectra }\left(M_{\mathrm{r}}\right)\end{array}$} & \multirow[b]{2}{*}{$\begin{array}{l}\text { Charge } \\
\text { state }^{a}\end{array}$} & \multicolumn{5}{|c|}{ Relative ion abundance } & \multirow[b]{2}{*}{$\mathrm{CE}$} \\
\hline & & $\begin{array}{l}\text { ES } \\
(\%)\end{array}$ & $\begin{array}{r}+S A \\
(\%)\end{array}$ & $\begin{array}{r}-S A \\
(\%)\end{array}$ & $\begin{array}{r}+\mathrm{CHCA} \\
(\%)\end{array}$ & $\begin{array}{r}-\mathrm{CHCA} \\
(\%)\end{array}$ & \\
\hline 6328.6 & $3(1)$ & 6 & - & - & - & - & + \\
\hline 6479.0 & $4(1)$ & 10 & - & - & 12 & 9 & + \\
\hline 6492.6 & $4(1)$ & 20 & - & 11 & 14 & 9 & + \\
\hline 6516.0 & $4(0)$ & 8 & $41^{b}$ & - & 16 & 8 & - \\
\hline 6542.0 & $3(1)$ & 5 & $41^{b}$ & - & - & - & - \\
\hline 6560.0 & & - & 21 & & 6 & 6 & \\
\hline 6610.0 & & - & 12 & 5 & 7 & 5 & - \\
\hline 6633.0 & & - & - & 7 & - & - & + \\
\hline 6658.5 & $4(0)$ & 4 & - & - & - & - & + \\
\hline 6683.0 & & - & - & - & 11 & 12 & - \\
\hline 6700.0 & & - & 37 & $15^{b}$ & 16 & 15 & - \\
\hline 6719.0 & & - & 51 & $22^{b}$ & - & 16 & - \\
\hline 6751.0 & & - & - & 16 & 10 & 11 & - \\
\hline 6773.8 & $3(0)$ & 4 & 24 & - & - & - & + \\
\hline 6823.0 & & - & 21 & 21 & - & - & - \\
\hline 6886.5 & $4(3)$ & 10 & 17 & - & 8 & 5 & - \\
\hline 6931.0 & & - & - & 12 & - & 5 & - \\
\hline 6944.7 & $4(0)$ & 12 & 10 & - & - & - & - \\
\hline 6966.0 & $3(0)$ & 2 & 9 & 11 & - & - & + \\
\hline 7086.2 & $3(0)$ & 4 & - & - & 0 & 4 & + \\
\hline 7116.3 & $4(1)$ & 3 & - & - & - & - & - \\
\hline 7143.6 & $4(0)$ & 18 & - & - & 7 & 3 & + \\
\hline 7240.9 & $3(0)$ & 6 & - & - & - & 4 & - \\
\hline 7437.6 & $3(2)$ & 5 & - & - & - & - & - \\
\hline 7501.5 & $3(1)$ & 4 & - & 9 & - & - & - \\
\hline 7517.2 & $3(1)$ & 7 & - & - & - & 15 & - \\
\hline 7533.0 & $5(4)$ & 100 & - & - & 31 & 16 & - \\
\hline 7555.9 & $3(1)$ & 10 & 16 & - & 30 & 15 & - \\
\hline 7572.7 & $5(1)$ & 18 & - & 19 & 28 & 16 & - \\
\hline 7587.2 & $5(2)$ & 18 & 19 & 20 & - & - & - \\
\hline 7630.0 & $3(1)$ & 35 & - & 14 & - & - & - \\
\hline 7663.0 & & - & - & 14 & 6 & - & - \\
\hline 7686.2 & $4(1)$ & 5 & 13 & - & - & 4 & - \\
\hline 7742.7 & $3(3)$ & 2 & - & 7 & - & - & - \\
\hline $13,183.3$ & $3(2)$ & 10 & - & - & - & 19 & - \\
\hline $13,277.9$ & $3(2)$ & 7 & - & - & 20 & 26 & - \\
\hline $13,391.9$ & $3(2)$ & 13 & - & - & 86 & 100 & - \\
\hline $13,415.2$ & $4(0)$ & 29 & - & - & - & 87 & - \\
\hline $13,432.8$ & $4(0)$ & 92 & 100 & - & 97 & 81 & - \\
\hline $13,458.5$ & $4(0)$ & 27 & - & 100 & 100 & 69 & - \\
\hline $13,532.0$ & $3(2)$ & 15 & 75 & 88 & - & - & - \\
\hline $13,558.4$ & $4(0)$ & 29 & - & - & 35 & 39 & - \\
\hline $13,640.0$ & & - & 47 & 53 & - & 14 & - \\
\hline $13,776.3$ & $3(3)$ & 4 & 22 & - & - & - & - \\
\hline $26,800.0$ & & - & - & - & 9 & 6 & - \\
\hline
\end{tabular}

a See Table 1 for explanation of charge state, +SA, - SA, +CHCA, and - CHCA. Species observed using one method but with no corroboration: ES $/ \mathrm{MS}-M_{r} 6198.3[3(2), 4 \%], M, 6254.0[3(1), 2 \%] ; M_{r}$ $6457.6[3(2), 13 \%] ; M_{r} 6589.8[4(3), 7 \%] ; M_{r} 7116.3[4(1), 3 \%] ; M, 7437.6[3(2), 5 \%] ; M_{r} 7653.7[4(2)$, $7 \%$ ]; $M_{r} 7730.1[3(1), 8 \%] ; M_{r} 8132.7[4(3), 3 \%] ; M_{r} 8851.6[4(4), 13 \%] ; M_{r} 13,367.9[3(0), 41 \%] ; M_{r}$ 13,482.8 [3(O), 15\%]. MALDI/TOF/MS - sinapinic acid (positive ion), $M_{r}$ 6810 (19\%), $M_{r} 13,880$ (12\%), $M_{r} 27,000(3 \%)$; sinapinic acid (negative ion), $M_{r} 6530(12 \%), M_{r} 6856(18 \%), M_{r} 6876(14 \%)$, $M_{\mathrm{r}} 6977$ (9\%), $M_{\mathrm{r}} 7004(8 \%), M_{\mathrm{r}} 7678(12 \%), M_{r} 7699(12 \%), M_{\mathrm{r}}$ 27, 160 (10\%); $\alpha$-cyano-4-hydroxycinnamic acid (positive ion), $M_{\mathrm{r}} 7362(6 \%), M_{\mathrm{r}} 7616(15 \%), M_{\mathrm{r}} 7786(6 \%)$;-cyano-4-hydroxycinnamic acid (negative ion), $M_{\mathrm{r}} 6844(7 \%), M_{\mathrm{r}} 6897(6 \%), M_{\mathrm{r}} 7185(4 \%), M_{\mathrm{r}} 7484(6 \%)$.

${ }^{b}$ lons are seen as shoulders on major peaks. 


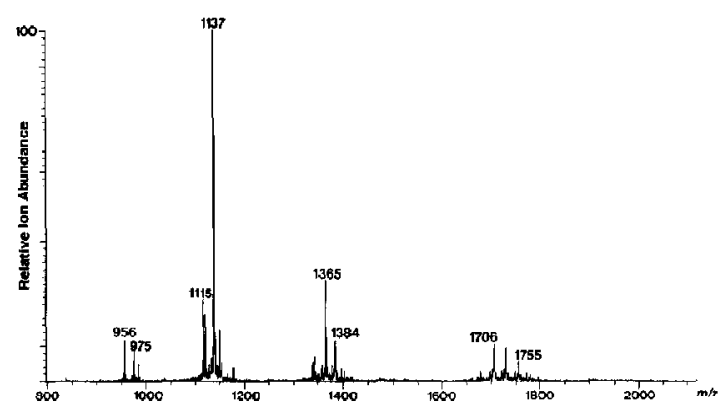

Figure 6. ES mass spectrum of the venom from $N$, mossambica.

ceps has been found to lack phospholipase $\mathrm{A}_{2}$ enzymes, and this has been postulated to be true of the other mambas [36]. Two phospholipase $\Lambda$ fractions extracted from the venom of $H$. haemachatus venom comprised $10 \%$ of the total venom [40]. A study on the venom of N. mossambica using LC with UV detection estimated the phospholipase $A_{2}$ content to be $33 \%$ $w / w$, and the venom of $N$. melanoleuca also contained a significant amount of phospholipase $A_{2}$, wilh plospholipase activity approximately five times that of $N$. mossambica [46]. Consequently, we expected the ES mass spectra of $H$. haemachatus (Figure 4), N. mossambica (Figure 6), and N. melanoleuca (Figure 7) to contain ions of significant abundance that would deconvolute to masses of approximately $13,000\left([\mathrm{M}+9 \mathrm{H}]^{9+}\right.$ species approximately $m / z \quad 1470-1500,[\mathrm{M}+8 \mathrm{H}]^{8+}$ species approximately $m / z \quad 1660-1690$, and $[\mathrm{M}+7 \mathrm{H}]^{7+}$ species approximately $m / z$ 1900-1930). The ES mass spectra of $H$. haemachatus and $N$. mossambica provide no indication of larger peptides, but the MALDI/TOF mass spectra of the venom from $H$. haemachatus, even when acquired at low laser powers to aid the detection and resolution of smaller molecular weight peptides, yielded some response for the phospholipase fraction, particularly when sinapinic acid matrix (positive ion) was used. Higher laser powers greatly increased the signal for these species.

The ES mass spectrum of $N$. melanoleuca indicated the presence of peptides of molecular masses 13,344 and 13,432 , but a truer measure of the relative abun-

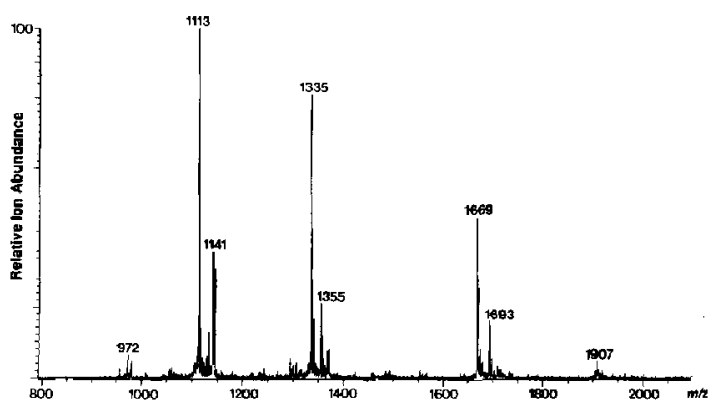

Figure 7. ES mass spectrum of the venom from $N$. melanoleuca.

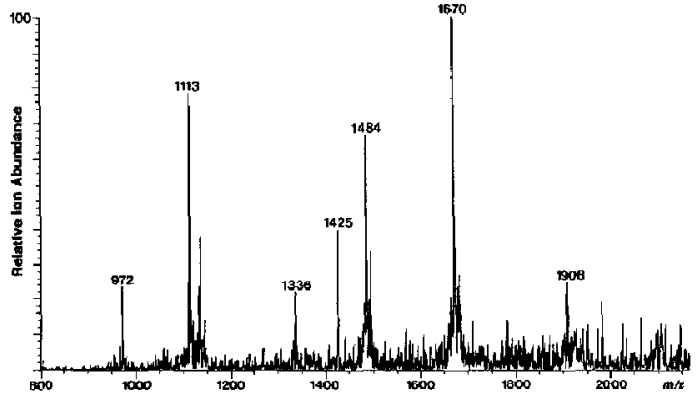

Figure 8. Summed ES mass spectrum obtained from $\mathrm{CE} / \mathrm{MS}$ of the venom from $N$. melanoleuca.

dances of peptides in the venom of $N$. melanoleuca was believed to be the total ES mass spectrum obtained after CE/MS, where the peptides in the venom were essentially admitted to the source one at a time, and it was found that the peptides of molecular weights of approximately $13,000 \mathrm{Da}$ had a much higher relative ion abundance (Figure 8). This was reflected by the unknown venom of $M$. fulvius, where the most abundant ions in the flow injection ES mass spectrum resulted from a peptide of molecular mass 7533 , with the other significant ions resulting from peptides of molecular masses of approximately 13,400. In the MALDI/TOF mass spectra of $M$. fulvius, whether sinapinic acid or $\alpha$-cyano-4-hydroxycinnamic acid was used as the matrix, the base peaks resulted from these larger peptides. This was also true of the totaled spectrum from CE/MS (Figure 9). These findings led to the conclusion that the responses for the higher molecular weight peptides were suppressed during flow injection ES/MS analysis.

\section{Ion Suppression in the MALDI / TOF Mass Spectra}

The MALDI/TOF mass spectra of the venom from $D$. polylepis revealed no significant ions above $m / z 9000$, and this was also seen to be apparent from the ES

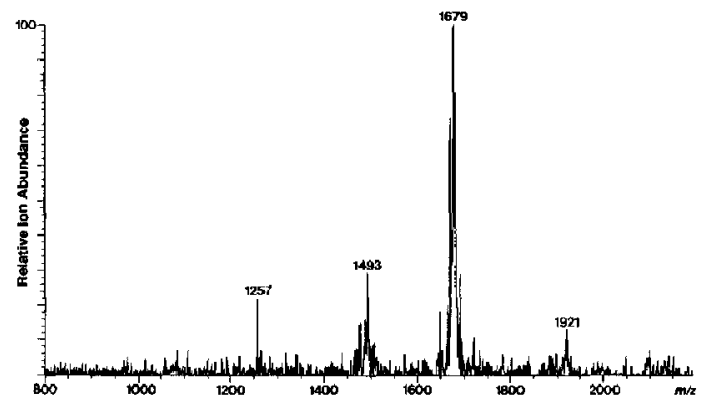

Figure 9. Summed ES mass spectrum obtained from $C E / M S$ of the venom from $M$. fulvius. 
spectrum (Figure 2, Table 2). The venom of $D$. polylepis has been roughly quantified; the major component of the venom, toxin $I\left(M_{r} 7132.4\right)$, constitutes $20 \%$ of the venom [49]. The ES spectrum is dominated by the $[\mathrm{M}+7 \mathrm{H}]^{7+}$ and $[\mathrm{M}+6 \mathrm{H}]^{6+}$ ions of toxin $\mathrm{I}$ at $\mathrm{m} / \mathrm{z}$ 1020 and 1190 , respectively, but it can be seen that the positive ion MALDI/TOF mass spectrum using sinapinic acid matrix is dominated, instead, by an ion of $m / z$ 6806, resulting from toxin $C(1.5 \%$ of venom) [49], whereas the base peak in the negative ion MALDI/TOF mass spectrum results from protein CM-3. The relative ion abundances of toxin $I$ in MALDI/TOF/MS using sinapinic acid matrix are $36 \%$ in the positive ion mode and $16 \%$ in the negative ion mode. The relative abundances of species seen in the ES spectrum have been further clarified by CE/ ES/SIMMS experiments, and other significant peptides in this venom that show only a small response include peptides of moleçular weights 7986 (toxin d) and 8004 $[47,48]$. This suppression of specific peptides can be overcome by the use of alternative matrices. The positive ion MALDI/TOF mass spectrum obtained using a-cyano-4-hydroxycinnamic acid as the matrix (Figure 2, Table 2) yielded the $[\mathrm{M}+\mathrm{HI}]^{+}$ion of toxin $\mathrm{I}$ as the base peak, together with a significant response for both toxin $\mathrm{d}$ and the peptide of molecular weight 8004 .

The dependency of the relative ion abundances on the matrix is also demonstrated by the MALDL/TOF mass spectra of the venom of $D$. jamesoni (Figure 1, Table 1). Using sinapinic acid as the matrix, the base peak in the spectrum is a peptide of molecular mass 6634, in agreement with the ES mass spectrum. Use of a-cyano-4-hydroxycinnamic acid yields the $[\mathrm{M}+\mathrm{H}]^{+}$ ion of toxin $\mathrm{S}_{5} \mathrm{C}_{4}$ as the base peak, with the peptide of molecular mass 6634 showing a relative ion abundance of $20 \%$. Furthermore, $\alpha$-cyano-4-hydroxycinnamic acid matrix also provides more information concerning peptides of molecular weights greater than $6900 \mathrm{Da}$ (Figure 1, Table 1). This selectivity is helpful where less abundant peptides yield larger responses than indicated by other methods, such as the peptides between $m / z 6820$ and $m / z 6950$ in the MALDI/TOF mass spectra of $D$. jamesoni, which were not seen using ES/MS, or the enhanced signal, seen using sinapinic acid, for peptides of molecular weights of approximately 7350,7400 , and $7670 \mathrm{Da}$ in the venom of $D$. polylepis relative to a-cyanw-4-lyydroxycinnamic acid. Another example is the $[\mathrm{M}+\mathrm{H}]^{+}$ion of toxin $\mathrm{F}_{8}$ from the venom of $D$. augusticeps. In the MALDI/TOF mass spectrum, using $\alpha$-cyano-4-hydroxycinnamic acid midtrix, it has a relative ion abundance of $94 \%$ relative to $8 \%$ in the ES mass spectrum but is not seen at all using sinapinic acid matrix.

Additional flexibility is obtained for MALDI/ TOF/MS by use of negative ionization. Toxins of molecular masses between $m / z 7200$ and 8000 were noted in the venom of $D$. angusticeps using sinapinic acid matrix in the positive ion mode; however, the relative response of these peptides was substantially increased by changing the polarity of the accelerating voltage (Figure 3 ).

\section{Molecular Mass Assignments of Sequenced Peptides}

The molecular mass assignments were used to provide confirmation of sequences reported in the literature (Tables 1-5). For most sequences, molecular masses are provided that agree with the sequences, but in some cases doubt exists where expected ions do not occur. Spectra of the full venom can definitely confirm the presence of a peptide of a particular molecular mass but cannot prove unequivocally that a toxin is not present because suppression was noted with both methodologies. For toxins in the molecular weight range $6000-9000 \mathrm{Da}$, lack of confirmation by either technique may suggest that the published sequence is incorrect, and mass analysis of the individual peptides would be desirable to unequivocally confirm the published sequences, particularly because peptides are observed with molecular masses that are close to the published sequences but do not match them (e.g., toxin IV, $H$. huemachatus). Obviously the possibility exists that some peptides may be suppressed using all methods.

A significant problem has been toxin $\mathrm{FS}_{2}$, which is the third most abundant peptide in the venom of $D$. polylepis. CE/ES/SIMMS experiments identified the presence of approximately 100 peptides in the venom of $D$. polylepis, but there was no indication of ions pertaining to a peptide of molecular mass 7018 [47]. It was thought, however, that an abundant peptide of molecular mass 7035 may be toxin $\mathrm{FS}_{2}$ [47], and positive ion MALDI/TOF/MS and ES/MS data would seem to confirm this; however, a peak at $\mathrm{m} / \mathrm{z} 7018$ was noted in the negative ion spectrum using $\alpha^{-}$ cyano-4-hydroxycinnamic acid matrix, albeit as a shoulder on the ion of $m / z 7035$, indicating the need for analysis of the extracted peptide.

\section{Conclusions}

Both ES/MS and MALDI/TOF/MS provide valuable information for the preliminary characterization of snake venoms. Where many closely related peptides are present, ES/MS is more useful because the resolution of MALDI/TOF/MS is too low to differentiate fully among them, and this can lead to the obscuring of some major componerits. MALDI/TOF/MS, however, is particularly useful for indicating the full range of peptides present because suppression of higher molecular weight peptides occurs during ES/MS. Suppression during MALDI/TOF/MS is more difficult to predict but can be overcome through the use of alternative matrices. The different information provided by both techniques indicates that to obtain as much preliminary data as possible about complex peptide mixtures, such as snake venoms, the use of both tech- 
niques to provide complementary and confirmatory information is desirable.

Inclusion of a separation step is vital for the complete characterization of snake venoms, particularly concerning minor components and multiple species of the same molecular weight. At present, interfacing LC or CE with ES/MS would seem to indicate the more complete approach.

\section{References}

1. Dole, M; Mack, L. L.; Hines, R. L.; Mobley, R. C.; Ferguson, L. D.; Alice, M. B. Chem. Phys. 1968, 49, 2240-2249.

2. Yamashita, M.; Fentr, J. B. J. Phys. Chem. 1984, 88, 4451.

3. Whitchouse, C. M.; Dreyer, R. N.; Yamashita, M.; Fenn, J. B. Anal. Chem. 1985, 57, 675 $\sim 679$.

4. Wong, S. F.; Meng, C. K.; Fenn, J. B. J. Phys. Chem. 1988, 92, 546-550.

5. Mann, M.; Meng, C. K.; Fenn, J. B. Anal. Chem. 1989, 61, 1702-1708.

6. Wong, S. F.; Meng, C. K.; Fenn, J. B. Proceetings of the 35th ASMS Conference on Mass Spectrometry and Allied Topics; Denver, CO; May 1987; pp. 33-34.

7. Meng, C. K; Mann, M.; Fenn, J. B. Proceedings of the 36th ASMS Canference on Mass Spectrometry and Allied Topics; San Francisco, CA; June 1988; pp. 771-772.

8. Chan, K. W. S.; Cook, K. D. Org. Mass Spectrom, 1984, 18, 423.

9. Pilosof, D.; Kim, H. Y.; Dyckes, D. Y.; Vestal, M. L. Anal. Chem. 1984, 56, 1236-1240.

10. Jonsson, G. P.; Hedin, A. B.; Hakansson, P. L.; Sundqvist, B. U. R.; Save, B. G. S.; Nielsen, P. F.; Roepstorff, P.; Johansson, K.-E.; Kamensky, I.; Lindberg, M. S. L. Anal. Chem. 1986, $58,1084-1087$.

11. Smith, R. D.; Loo, J. A.; Ogorzalek-Loo, R. R.; Busman, M.; Udseth, H. R. Mass Spectrum. Rev. 1991, 10, 359-451.

12. Fenn, J. B.; Mann, M.; Meng, C. K.; Wong, S. F.; Whitehouse, C. M. Mass Spectrom. Rev. 1990, 9, 37-70.

13. Huang, E. C.; Wachs, T.; Conboy, J. J.; Henion, J. D. Anal. Chem. 1990, 62, 713A-725A.

14. Bruins, A. P. Mass Spectrom. Rev. 1991, 10, 53.

15. Mann, M. Org. Mass Spectrom. 1990, 25, 575 .

16. Wiley, W. C.; McLaren, I. H. Rev. Sci. Instrum. 1955, 26, 1150.

17. Cotter, R. J. I. Anal. Chem. 1992, 64, 1027A-1039A.

18. Karas, K.; Hillenkamp, F. Anal. Chem. 1988, 60, 2299-2301.

19. Tanaka, K.; Waki, H.; Ido, Y.; Akita, S.; Yoshida, Y.; Yoshida, T. Rapid Commurt. Mass Spectrom. 1988, 2, 151.

20. Beavis, R. C.; Chait, B. T. Anal. Chem. 1990, 62, 1836-1840.

21. Karas, M.; Bahr, U.; Giessmann, U. Mass Spectrom. Reo. 1991, $10,335$.

22. Karas, M.r Bahr, U. Trends Anal. Chem. 1990, 9, 321.

23. Hillenkamp, F.; Karas, M.; Beavis, R. C.; Chait, B. T. Anal. Chem. 1991, 63, 1193A-1202A.
24. Tu, A. T. Venoms: Chemistry and Molecular Biology; Wiley: New York, 1977.

25. Lee, C. Y., Ed. Snake Venoms; Springer-Verlag: New York, 1979.

26. Viljoen, C. C.; Botes; D. P. J. Biol. Chem. 1974, 249, 366-372.

27. Dufton, M. J. Eur. I. Biochem. 1985, 153, 647-654.

28. Joubert, F. J.; Taljaard, N. Biochim. Biophys. Acta 1980, 623, 449-456.

29. Strydom, A. J. C. Biochim. Biophys. Acta 1973, 328, 491-509.

30. Joubert, F. J. int. J. Biochem. 1985, 17, 695-699.

31. Strydom, D. J. Syst. Zool. 1973, 22, 596-608.

32. Joubert, F. J.; Strydom, D. J. Hoppe-Seylers Z. Physiol Chem. 1980, 361, 1787-1794

33. Joubert, F. J.; Taljaard, N. Biochim. Biophys. Acta 1979, 579, 228-233.

34. Joubert, F. J.; Taljaard, N. Int. J. Biochem. 1980, 12, 567-574.

35. Joubert, F. J.; Viljoen, C. C. Hoppe-Seylers Z. Physiol. Chem. 1979, 360, 1075-1090.

36. Karlsson, E; Mbugua, P. M; Rodriguez-Ithurralde, D. I. Physiul. Puris 1984, 79, 232-240.

37. Karlsson, E.; Risinger, C.; Jolkkonen, M.; Wernstedt, C.; Adem, A. Taxicon 1991, 29, 521-526.

38. Joubert, F. J. Fur. J. Riochem. 1977, 74, 387-396.

39. Strydom, A. J. C.; Botes, D. P. I. Biol. Chem. 1971, 246, 1341-1349.

40. Joubert, F. J. Eur. J. Biochem. 1975, 52, 539-554.

41. Keller, R. M.; Baumann, R.; Hunziker-Kwik, E.-H.; Joubert, F. J.; Wuthrich, K. J. Mol. Biol. 1983, 163, 623-646.

42. Arseniev, A. S.; Wider, G.; Joubert, F. J.; Wuthrich, K.J. Mol. Biol. 1982, 158, 323-351.

43. Labhardt, A. M.; Hunziker-Kwik, E.-H.; Wuthrich, K. Eur. J. Biochem. 1988, 177, 295-305.

44. Feng, R: Yuan, 7. Procepdings of the 38th ASMS Conference on Mass Spectrometry and Allied Topics; Tucson, AZ, 1990; Pp. 998-999.

45. Sweetman, G. M. A.; Garner, G. V.; Tetler, L.; Theakston, R. D. G.; Gordon, D. B. Proceedings of the 19th Annual Meting of the British Mass Spectrometry Society; St. Andrews, UK, September 1992; p. 152.

46. Bougis, Г. E.; Marchot, P.; Rochat, H. Biochemistry 1986, 25, $7235-7243$.

47. Perkins, J. R.; Parker, C. E;; Tomer, K. B. Electrophoresis, in press.

48. Perkins, J. R.; Parker, C. E.; McGown, S. R,; Tomer, K. B. Proceedings of the 40th ASMS Conference on Mass Spectrometry and Allied Topics; Washington, DC, May 31-June 5, 1992; pp. 961-962.

49. Schweitz, H.; Bidard, J.-N.; Lazdunski, M. Toxicon 1990, 28 $847-856$.

50. Deterding, L. J; Moseley, M. A.; Tomer, K. B.; Jorgenson, J. W. Anal. Chem. 1989, 61, 2504.

51. Moseley, M. A.; Deterding, L. J.; Tomer, K. B.; Jorgenson, I. W. Rapid Commun. Mass Spectrom. 1989, 3, 87-93. 